\title{
Usefulness of Pressure-Sensitive Adhesives as a Pretreatment Material before Application of Topical Drug Formulations and a Peeling Tape for Excess Stratum Corneum Layers
}

\author{
Keisuke Kikuchi, Hiroaki Todo, and Kenji Sugibayashi* \\ Faculty of Pharmaceutical Sciences, Josai University; 1-1 Keyakidai, Sakado, Saitama 350-0295, Japan.
}

Received January 25, 2014; accepted March 11, 2014

\begin{abstract}
Two unique pressure-sensitive adhesive (PSA) tapes (PSA-A, -B) with different adhesive properties of commercial PSAs were prepared and evaluated for their usefulness as a pretreatment material prior to the application of transdermal therapeutic systems or topical drug formulations and also as a peeling agent against excess layers of the stratum corneum. In the present study, in vitro permeation experiments were conducted using vertical type diffusion cells and excised hairless rat or porcine skin from which the stratum corneum had been stripped several times with PSAs. The results obtained revealed that PSA-A and -B had higher stripping or peeling effects than those of the marketed PSAs. Marked changes were observed in skin barrier function before and after stripping using PSAs, and the enhancement effect on the skin permeation of drugs achieved by stripping the stratum corneum was markedly different between the PSAs. PSA-A, in particular, markedly improved skin permeation and the skin concentration of topically applied chemical compounds because it removed many layers of the stratum corneum when skin was stripped only a few times. In contrast, when PSA-B was used to pretreat the skin surface, the extent of skin permeation and concentration of drugs was safely increased because only a few layers of the stratum corneum were removed, even with repeated stripping. The enhancement effect achieved by PSA-B was not as high as that by PSA-A. Thus, stripping with PSA-A can be used as a penetration enhancement tool, whereas PSA-B can be used as a peeling material against excess layers of the stratum corneum.
\end{abstract}

Key words pressure-sensitive adhesive; pretreatment material; skin permeation; tape-stripping; peeling

One of the biggest limitations of transdermal drug delivery systems and topical drug formulations is the extremely low skin permeation of drugs immediately following their topical application. For example, inadequate pain relief against a needle injection was reported immediately after the application of a lidocaine patch. ${ }^{1)}$ The low permeation of drugs in the skin has been attributed to the strong barrier function of its uppermost layer, the stratum corneum, and the resulting long lag-time for drug diffusion through this layer. Corneocytes, together with the intercellular region in the stratum corneum, have been shown to function as a very compact and extensively lipophilic barrier; therefore, water evaporation from the body as well as the penetration of exogenous compounds into the body through the skin are very limited. ${ }^{2-4)}$ The lagtime of low molecular compounds may be several tens of minutes to several hours before steady-state flux is achieved. Thus, increasing the permeability of the stratum corneum barrier for the rapid onset of the pharmacological action of topically applied compounds is desired. Previous studies have focused on achieving high drug concentrations in the skin as well as increasing skin permeation in order to obtain adequate therapeutic effects. ${ }^{5,6)}$ Prodrug approaches and the application of chemical enhancers have been broadly utilized to enhance the skin penetration of topically applied drugs. In addition, physical means such as iontophoresis, electroporation, and microneedle arrays have recently been used as novel skin penetration-enhancing tools. ${ }^{7-12)}$ However, these physical means can be cumbersome due to their complicated systems. A simple shape and easy usage are two important conditions for the practical use of topical formulations.

In the present study, we pretreated the primary skin barrier, the stratum corneum, with a pressure-sensitive adhesive, PSA, without entrapping therapeutic drugs. PSAs have been widely used in in vitro and in vivo skin permeation studies to strip or peel off one to several layers of the stratum corneum. Tape stripping the stratum corneum, a procedure referred to as the dermatopharmacokinetic (DPK) method, has been previously used to evaluate the bioequivalence of topical drug formulations. However, the effectiveness of conventional PSA tapes was shown to be negligible when they were used to strip the skin once or twice because of their low stripping or peeling effect against the stratum corneum barrier. In addition, variability in the amount of the stratum corneum removed by stripping with PSAs may cause scattered skin permeation and pharmacological effects of topically applied drugs.

We prepared new PSA (PSA-A, -B) tapes with adequate adhesive properties by modifying the composition and preparation methods of conventional PSA cellophane tapes such as Cellotape ${ }^{\circledR}$ or Scotch Tape. Firstly, the adhesive properties of PSAs and commercial PSAs currently used were evaluated by the $180^{\circ}$ peeling, prove tack, and ball tack methods. ${ }^{13)}$ The effect of the number of times stripped with PSA-A and -B on the barrier function of hairless rat dorsal skin and porcine ear skin was evaluated by transepidermal water loss (TEWL), skin impedance, amount of the stratum corneum stripped, and morphological evaluation of skin sections. ${ }^{14)}$ In addition, the penetration-enhancement effect of tape-stripping with PSA-A and $-\mathrm{B}$ was evaluated on the skin permeation of lidocaine using in vitro skin permeation experiments following the application of its aqueous solution or tape formulation. The marketed PSAs, Cellotape ${ }^{\circledR}$ and Scotch $^{\circledR}$ were examined and compared with the newly prepared PSAs, PSA-A and -B.

The authors declare no conflict of interest. 


\section{Experimental}

Materials Lidocaine hydrochloride monohydrate was purchased from Sigma-Aldrich Japan (Tokyo, Japan). The Lidocaine Patch (Penless ${ }^{\circledR}$ ) was supplied by Maruho Co., Ltd. (Osaka, Japan). Other reagents and solvents were of reagent grade or HPLC grade and were used without further purification.

The preparation of PSA-A and -B was carried out at Lintec Corporation (Tokyo, Japan). The raw materials used to make these PSAs; $N$-vinyl-2-pyrrolidone (NVP), 2-ethyl hexyl acrylate (2EHA), and acrylic acid (AA), were obtained from Tokyo Chemical Industry Co., Ltd. (Tokyo, Japan). A cellophane membrane and PET film were used as the backing and liner, respectively, for both PSA-A and -B. Two kinds of PSA cellophane tapes; Cellotape ${ }^{\circledR}$ (Packaging tape, Series 405) and Scotch Tape (Scotch ${ }^{\circledR}$ packaging tape, Series 375SN), were obtained from Nichiban Co., Ltd. (Tokyo, Japan) and Sumitomo 3M, Ltd. (Tokyo, Japan), respectively.

Preparation of PSA Polymer mixtures and oligomer mixtures were prepared prior to the fabrication of PSA-A and -B. The compositions (molar ratio) of the polymer mixtures a and $b$ and oligomer mixture for PSA-A were 2EHA/AA=80/20, $2 \mathrm{EHA} / \mathrm{NVP}=88 / 12$, and $2 \mathrm{EHA} / \mathrm{NVP}=88 / 12$, respectively, and those for PSA-B were 2EHA/AA=82/18, 2EHA/NVP= $88 / 12$ and $2 \mathrm{EHA} / \mathrm{NVP}=80 / 20$, respectively (Table 1). All materials shown in Table 1 were mixed and dissolved in ethyl acetate. The resulting mixture was then coated on a sheet of cellophane backing layer and the solvent was dried to obtain PSA-A and -B. The resulting thickness of both PSA-A and -B was almost the same to the marketed PSAs.

Measurement of the Adhesive Properties of PSAs ${ }^{13)}$ The peeling force at an angle of $180^{\circ}$ was determined by an Autograph (AG-IS, Shimadzu, Kyoto, Japan) as an adhesive property of PSAs. Test PSAs $25 \mathrm{~mm}$ in width and $70 \mathrm{~mm}$ in length were adhered to a test board by two reciprocations at a rate of $10 \mathrm{~mm} / \mathrm{s}$ using a pressed roller weighing $2 \mathrm{~kg}$. This adhesive property was measured at a temperature of $23^{\circ} \mathrm{C}$ and relative humidity of $50 \%$. Three determinations were performed. This procedure was followed by the Japanese Industrial Standard (JIS) method.
A probe tack tester (RPT100, Rhesca Corporation Ltd., Hino, Tokyo, Japan) was also used to determine another adhesive force of PSAs. A polished stainless probe with a diameter of $18 \mathrm{~mm}$ was used, and PSA samples were adhered to the probe at a pressure of $100 \mathrm{~g} / \mathrm{cm}^{2}$ for $1 \mathrm{~s}$. The maximum adhered force $\left(\mathrm{g} / \mathrm{cm}^{2}\right)$ was determined in the probe at $23^{\circ} \mathrm{C}$ and a relative humidity of $50 \%$. Nine determinations were used to calculate the mean value.

The ball tack method was performed by a tester (PI-1201, Tester Sangyo Co., Ltd., Miyoshi, Saitama, Japan) using 32 kinds of test balls (SUJ2) with diameters of $1 / 32$ to 1 inch. These balls were washed with toluene and ethanol prior to measurements. PSA samples were $50 \mathrm{~mm}$ in width and $250 \mathrm{~mm}$ in length. The slope and running length were set at $30^{\circ}$ and $100 \mathrm{~mm}$, respectively. The measurement position was set at $100 \mathrm{~mm}$ from the bottom of the running plate. The ball tack method was conducted at the same temperature and humidity as those used in the $180^{\circ}$ peel and probe tack methods. The results were shown by the ball number. The average value of three determinations was calculated.

Experimental Animals The WBN/ILA-Ht strain of male hairless rats, weighing between 200 and $230 \mathrm{~g}$ b.w., were obtained from the Life Science Research Center, Josai University (Sakado, Saitama, Japan) and Ishikawa Experimental Animal Laboratories (Fukaya, Saitama, Japan). Rats were bred in a room maintained at $25 \pm 2^{\circ} \mathrm{C}$, in which the on and off times of the light were 07:00 and 19:00, respectively. Animals had free access to water and food (MF, Oriental Yeast Co., Ltd., Tokyo, Japan).

Porcine ears were obtained from Saitama Experimental Animal Supply (Sugito, Saitama, Japan) and frozen until use.

Animal experiments were performed after obtaining the permission of the Animal Experiment Committee of Josai University (Sakado, Saitama, Japan).

Single Stripping Test from the Stratum Corneum Surface Using PSA Hairless rats were anesthetized with an intraperitoneal (i.p.) injection of pentobarbital $(50 \mathrm{mg} / \mathrm{kg})$, the right and left dorsal skin was shaved, and several layers of the stratum corneum $\left(4 \mathrm{~cm}^{2}\right.$, two regions each) were stripped using different kinds of PSAs. PSAs were adhered to the skin

Table 1. Recipe to Fabricate PSA-A and -B

\begin{tabular}{lcccc}
\hline \hline (1) PSA-A & & & & \\
\hline Material & Conc. $(\%)$ & Composition ratio & Molar ratio & $\left.M_{\mathrm{n}}{ }^{a}\right)(\mathrm{kD})$ \\
\hline Polymer mixture a & 35 & 100 & 2EHA/AA=80/20 & $c a .650$ \\
Polymer mixture b & 45 & 155 & 2EHA/NVP=88/12 & $c a .800$ \\
Oligomer mixture & 90 & 115 & 2EHA/NVP. $=88 / 12$ & \\
Glycerin & 100 & 70 & & \\
Isothianate cross-linker & 50 & 200 & & \\
Ethyl acetate & 0 & 1 & & \\
\hline
\end{tabular}

(2) PSA-B

\begin{tabular}{lcccl}
\hline Material & Conc. $(\%)$ & Composition ratio & Molar ratio & $M_{\mathrm{n}}^{a)}(\mathrm{kD})$ \\
\hline Polymer mixture a & 35 & 100 & 2EHA/AA $=82 / 18$ & $c a .1000$ \\
Polymer mixture b & 45 & 155 & 2EHA/NVP=88/12 & $c a .800$ \\
Oligomer & 85 & 123 & $2 \mathrm{EHA} / \mathrm{NVP}=80 / 20$ & \\
Isothianate cross-linker & 50 & 1 & & \\
Ethyl acetate & 0 & 300 & & \\
\hline
\end{tabular}

a) Number represents the average molecular weight. 
surface in tape stripping procedure with the load of $100 \mathrm{~g} / \mathrm{cm}^{2}$ with a thumb.

The effect of stripping with PSAs on the barrier function of the stratum corneum was determined in detail using the three following methods. First, the stratum corneum was removed and its weight was determined following successive tape stripping, one to 20 times, with PSAs. PSA weights were measured before and after the skin had been stripped using a microbalance (MX5, Mettler-Toledo K.K., Tokyo, Japan). The weight of the stratum corneum removed was used as an index of the peeling force of the PSA. Second, transepidermal water loss (TEWL) from hairless rat dorsal skin was determined by a Vapo Scan (AS-VT100RS, Asahi Techno Lab., Yokohama, Japan) during successive tape stripping (one to 20 times). Third, skin impedance was also determined during successive tape stripping as follows: hairless rat dorsal skin was excised after tape stripping and placed between side-by-side diffusion cells (with an effective diffusional area of $0.95 \mathrm{~cm}^{2}$ ), ${ }^{15}$ ) and phosphate buffered saline (PBS), $\mathrm{pH} 7.4$, was added to both the cells for $2 \mathrm{~h}$. An $\mathrm{Ag}$ anode or $\mathrm{AgCl}$ cathode electrode was set facing the dermis or stratum side, respectively, in the cells and skin impedance was determined using a $10 \mathrm{~Hz}$ alternative current (AC) by an Impedance Meter (Advance, Tokyo, Japan).

Preparation and Observation of Skin Pieces Excised hairless rat dorsal skin taken from the diffusion cell after the in vitro skin permeation experiment was cut into $2 \mathrm{~mm}$-thick pieces by a double-sided blade razor S (Feather Safety Razor Co., Ltd., Osaka, Japan), occluded in cryoembedding agent for the Kawamoto method (SCEM, Leica Microsystems Co., Ltd., Tokyo, Japan), and frozen in isopentane cooled with dry ice. A vertical slice of the skin $(10 \mu \mathrm{m}$ thick) was prepared by a cryostat (CM3050S, Leica Microsystems Co., Ltd.). The prepared skin slice was stained with hematoxylin and eosin and observed under a microscope (IX71, Olympus, Tokyo, Japan).

In Vitro Skin Permeation Experiment ${ }^{16-19)}$ Two pieces each from the right and left dorsal skin of hairless rats were shaved and excised under anesthesia by an i.p. injection of pentobarbital. In addition, stripped skin was excised from rats after the stratum corneum had been stripped 20 times by Cellotape ${ }^{\circledR}$. After fat and blood had been removed from the dermis, intact or stripped skin was set in a Franz type diffusion cell (receiver cell volume, $6.0 \mathrm{~mL}$; and effective diffusion area, $\left.1.77 \mathrm{~cm}^{2}\right){ }^{20)}$ After $1 \mathrm{~mL}$ of PBS had been added to the stratum corneum side for $1 \mathrm{~h}$ to hydrate the skin, it was replaced with the same volume of $10 \mathrm{~mm}$ lidocaine in PBS to start the skin permeation experiment. Diffusion cells were maintained at $32^{\circ} \mathrm{C}$ and the receiver solution was agitated at $500 \mathrm{rpm}$ by a star-head stir bar and magnetic stirrer. Aliquots $(0.5 \mathrm{~mL})$ were periodically sampled, with the same volume of PBS being added to keep the volume constant. The concentration of lidocaine in the sample was determined by HPLC.

In addition, skin that had been stripped 5 or 20 times by PSA-A, -B, or Cellotape was excised and placed in the Franz type cell, and the marketed lidocaine patch (lidocaine amount, $1.82 \mathrm{mg} / \mathrm{cm}^{2}$ ) was applied. The following permeation experiments were performed using the same procedure as that for the solution application experiments.

Determination of the Skin Concentration of Lidocaine Hairless rat dorsal skin samples were obtained after the in vitro skin permeation experiment, washed with PBS, and cut into an effective diffusion area to weigh the sample. A speci- men was cut into pieces using scissors, suspended in $1 \mathrm{~mL}$ of ethanol, and incubated for $24 \mathrm{~h}$ to extract lidocaine. The amount of lidocaine extracted was determined by HPLC.

Additionally, a piece of skin was immersed in PBS solution containing lidocaine $(A \mathrm{mg})$, and the amount of lidocaine $(B \mathrm{mg})$ in the skin was subsequently determined by adding ethanol using the same method as above. The amount of lidocaine $(C \mathrm{mg})$ in the solution was measured after the skin was removed. The extraction ratio was determined as $B /(A-C)$.

Determination of Lidocaine Ethylparaben (EB) was used as an internal standard in the quantitative determination of lidocaine. Thus, the lidocaine solution and acetonitrile $(1: 1)$ were mixed together and centrifuged $(15000 \times \mathrm{rpm}$, $5 \mathrm{~min}, 4^{\circ} \mathrm{C}$ ) to obtain supernatant for the HPLC assay. The HPLC system consisted of a pump (LC-20AD), UV detector (PD-20A), system controller (SCL-10A ${ }^{\mathrm{VP}}$ ), auto injector (SIL20A), degasser (DGU-20A A $_{3}$, column oven (CTO-20A), and analysis software (LC solution) (All from Shimadzu, Kyoto, Japan). The column was Inertsil ${ }^{\circledR}$ ODS-3 $5 \mu \mathrm{m}, 4.6 \times 150 \mathrm{~mm}$ (GL Sciences Inc., Tokyo, Japan), and the temperature was adjusted at $40^{\circ} \mathrm{C}$. The mobile phase was $0.1 \%$ distilled water adjusted to $\mathrm{pH} 2.5$ by phosphoric acid:acetonitrile $=7: 3+5 \mathrm{~mm}$ 1-heptanesulphonate. The injection volume was $20 \mu \mathrm{L}$ and detection was performed at UV $260 \mathrm{~nm}$.

Statistical Analysis Statistical analyses for the amount of stratum corneum stripped by the different kinds of PSAs and the lidocaine concentration in the skin were performed using ANOVA with nonrepeated measures, and $p<0.05$ was considered significant.

\section{Results}

Adhesive Properties of Several PSAs Table 2 summarizes the adhesive properties of PSAs determined by the three methods; $180^{\circ}$ peel, probe tack, and ball tack. The peel force for PSA-B was lowest, while those for the other PSAs were in the following order; Cellotape $<$ PSA-A $<$ Scotch Tape. In addition, the probe tack was markedly lower for PSA-B than for the other PSAs, which was similar to the results obtained for the peel force. However, the probe tack was similar between PSA-A, Cellotape, and Scotch Tape. The ball tack of PSA-B had the highest adhesive force, while those for the other PSAs were in the following order; Scotch Tape $<$ Cellotape tape $<$ PSA-A. The highest and lowest adhesive forces observed using the ball tack method were the same as in measured in the $180^{\circ}$ peel method.

Weight of the Stratum Corneum Removed by PSA Stripping Figure 1 shows the weight of the stratum corneum removed by stripping hairless rat dorsal skin and pig ear skin one time with different kinds of PSAs. The adhesive force estimated by the amount of stratum corneum removed $\left(\mu \mathrm{g} / \mathrm{cm}^{2}\right)$ from both skin types was in the order of PSA-A $>$ PSA-B $\gg$ marketed PSA cellophane tapes (Cellotape

Table 2. Adhesive Properties of the Various PSAs

\begin{tabular}{lccc}
\hline \hline PSA & $\begin{array}{c}180^{\circ} \text { peel } \\
(\mathrm{N} / 25 \mathrm{~mm})\end{array}$ & $\begin{array}{c}\text { Prove tack } \\
(\mathrm{g} / 18 \phi)\end{array}$ & $\begin{array}{c}\text { Ball tack } \\
(\text { No. })\end{array}$ \\
\hline PSA-A & 7.6 & 846 & 16 \\
PSA-B & 2.5 & 418 & 27 \\
Cellotape ${ }^{\circledR}$ & 5.8 & 958 & 11 \\
Scotch Tape $^{\circledR}$ & 11.6 & 903 & 5 \\
\hline
\end{tabular}


and Scotch Tape). No significant differences were observed in the results obtained for the two marketed PSA tapes. The adhesive forces against the stratum corneum were significantly higher with PSA-A and -B than with Cellotape $(p<0.01)$. Interestingly, the amount of the stratum corneum removed from hairless rat skin by stripping one time was approximately twice that from pig skin.

The relationship between the number of times stripped using PSAs and the cumulative amount of the stratum corneum removed from hairless rat dorsal skin was determined. Figure 2 shows the results obtained for the 4 PSAs. When PSA-A was used, the amount of the stratum corneum removed markedly increased from the first to the third strips. However, only a small amount of the stratum corneum was removed after skin was stripped four times or more. In addition, the cumulative amount of the stratum corneum removed from the first to the third strip using PSA-A was similar to that removed when skin was stripped 10 times with Cellotape or Scotch Tape. In contrast, the amount of the stratum corneum removed by the first strip was very high when PSA-B was used, which was similar to PSA-A; however, subsequent stripping only removed a small amount of the stratum corneum.

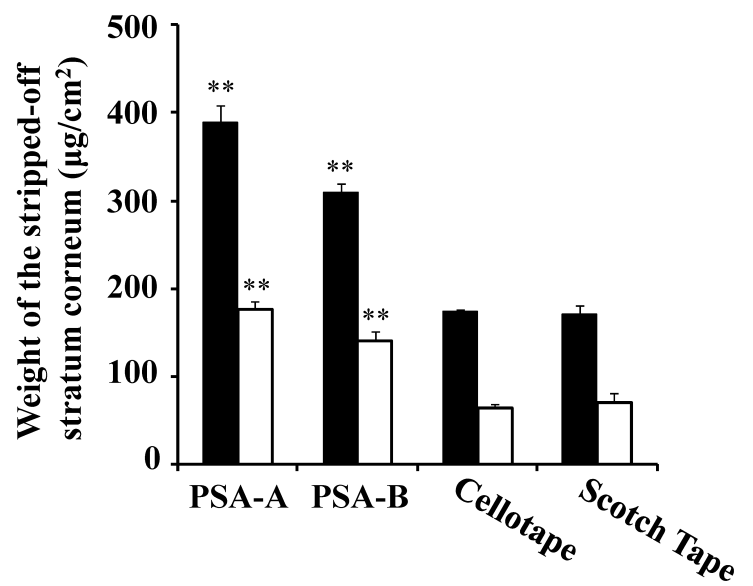

Fig. 1. Weight of the Stratum Corneum Removed by Stripping Hairless Rat Skin One Time with Different PSAs

Symbols: $\square$ : Hairless rat dorsal skin, $\square$ : Pig ear skin. Each value shows the mean \pm S.E. $(n=3)$. Statistical analysis was performed by ANOVA with nonrepeated measures. $* * p<0.01$.
The amount of the stratum corneum removed when skin was stripped 10 times was similar between PSA-A, Cellotape, and Scotch Tape, but was markedly lower with PSA-B.

These results indicate that PSA-A has a higher stripping effect than that of the commercial PSAs, Cellotape and Scotch Tape, and only a small amount of the stratum corneum was removed when skin was stripped four or more times.

Effect of PSA Stripping on TEWL and Skin Impedance Tape stripping clearly decreases the skin's barrier function. Since TEWL and skin impedance are good indices of the skin's barrier function, the effect of the number of times the skin was stripped on the barrier function of skin was determined using these indices. Figure 3 shows the results obtained. TEWL versus the tape-stripping profile (Fig. 3a) was similar to the reciprocal of skin impedance versus the tapestripping profile (Fig. 3b). TEWL and the reciprocal of skin impedance markedly increased when skin was stripped from 1 to 4 times with PSA-A, and remained constant from the fourth strip. PSA-A had a stronger effect on TEWL and skin impedance than that of the commercial PSAs. On the other hand, although PSA-B had a marked effect on these indices, especially with the first strip, subsequent stripping had a negligible

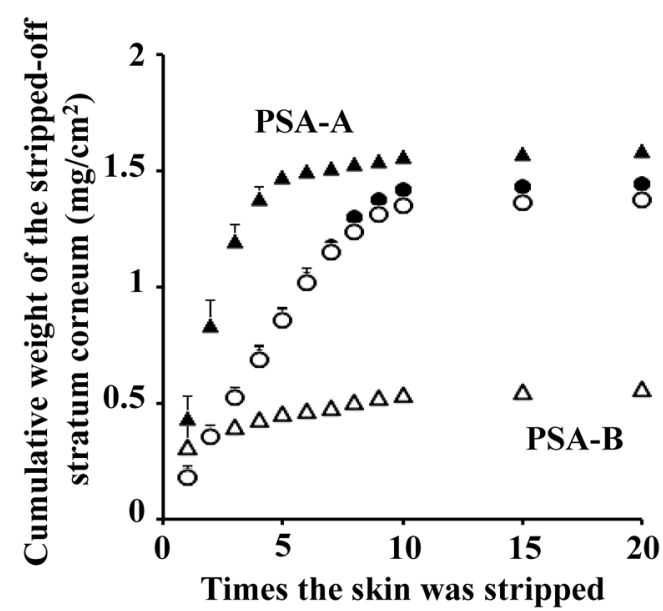

Fig. 2. Effect of the Number of Times Skin Was Stripped on the Cumulative Weight of the Stratum Corneum Removed from Hairless Rat Skin

Symbols: $\boldsymbol{\Delta}$ : PSA-A, $\triangle$ : PSA-B, $\bullet$ : Cellotape $^{\circledR}, \bigcirc$ : Scotch Tape. Each value shows the mean \pm S.E. $(n=3)$.
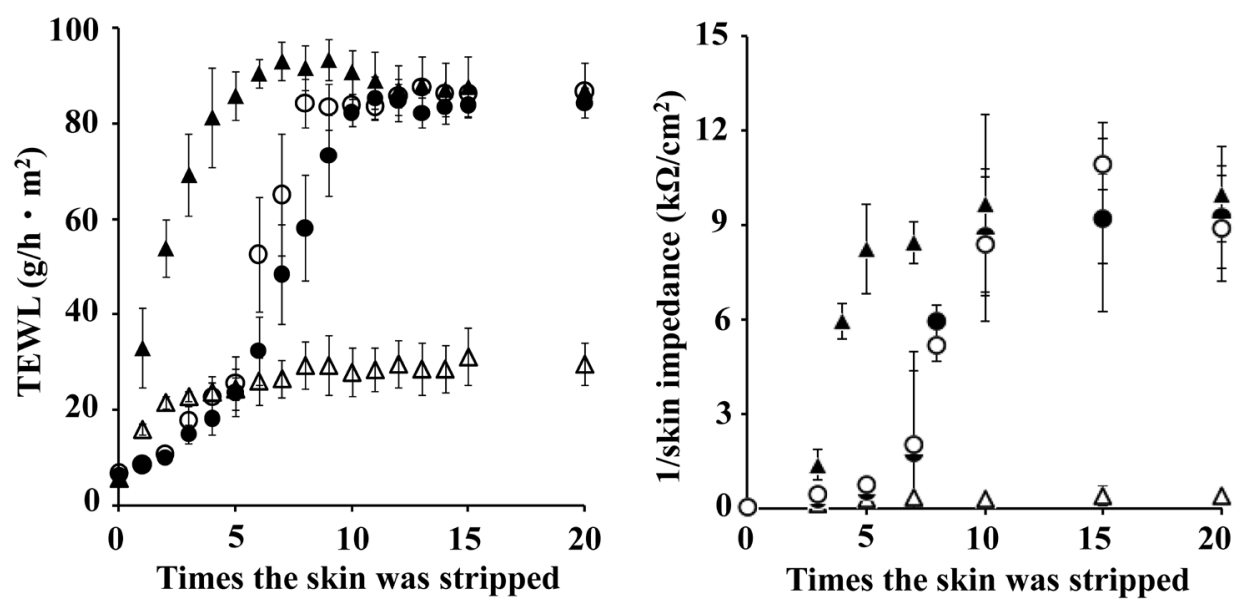

Fig. 3. Effect of the Number of Times Skin Was Stripped on Transepidermal Water Loss (TEWL) and Skin Impedance Values Symbols: $\boldsymbol{\Delta}$ : PSA-A, $\triangle$ : PSA-B, ๑: Cellotape $^{\circledR}$, $\bigcirc$ : Scotch Tape. Each value shows the mean \pm S.E. $(n=3-5)$. 
effect. Thus, the effect of stripping the skin two or more times on these indices was lower with PSA-B than with other PSAs. A similar constant stripping effect was observed with Cellotape and Scotch Tape when skin was stripped 1 to 10 times. These results were consistent with those shown in Fig. 2.

Observation of Skin Morphology Figures $4 \mathrm{a}$ and $\mathrm{b}$ show $\mathrm{H}-\mathrm{E}$ stained hairless rat dorsal intact and stripped skin sections, respectively, following in vitro skin permeation experiments. These skin sections had been stripped 20 times with Cellotape. Intact skin was covered by the stratum corneum, consisting of several layers of corneocytes, whereas stripped skin was not. Figures $4 \mathrm{c}-\mathrm{g}$ and $\mathrm{g}-\mathrm{j}$ show cross-sections of skin slices that had been stripped 5 and 10 times, respectively, with different types of PSAs. Stripping the skin five and 10 times with PSA-A (c, g) or 10 times with Cellotape (i) and Scotch Tape (j) fully removed the stratum corneum, whereas several layers of the stratum corneum remained following stripping 5 or 10 times with PSA-B $(\mathrm{d}, \mathrm{h})$. These results indicate that PSA-A had very strong adhesive properties against the stratum corneum, such that it was fully removed after stripping was performed a few times. In contrast, PSA-B could not remove all layers of the stratum corneum, even when skin was stripped several times.

In Vitro Skin Permeation of Lidocaine Figure 5 shows the time courses of the cumulative amount of lidocaine that permeated from its solution through hairless rat dorsal skin. Several stripping pretreatments by PSAs were performed prior to the application of lidocaine solution. The cumulative amount permeated over $6 \mathrm{~h}$ was as follows: the skin permeation of lidocaine was 5.2, 9.2, or 22 times higher than that through intact skin (no stripping) when skin was stripped one, two, or three times with PSA-A, respectively. Skin permeation was only 2 to 4 times higher when skin was stripped one to 20 times with PSA-B. On the other hand, skin permeability with tape stripping using the marketed cellophane tapes, Cellotape or Scotch Tape, was 1 to 10 times higher than that without stripping, but plateaued after skin had been stripped 10 to 20 times. Skin permeability was 25 - or 29 -fold higher than that without tape stripping when skin had been stripped ten times with Cellotape or Scotch Tape, respectively. These results suggest that PSA-A and -B have different skin permeation enhancement effects than those of the marketed PSAs due to their stripping abilities against the stratum corneum.

The relationship between the barrier function of the skin and skin permeation profiles of lidocaine was evaluated with different PSA treatments. Figure 6 shows the cumulative amount of lidocaine that permeated through the skin over $6 \mathrm{~h}$ in the axis of the ordinate and TEWL (a), reciprocal of skin impedance (b), and the cumulative amount of the stratum corneum removed (c) by tape stripping in the axis of the abscissa. Interestingly, markedly similar profiles were observed among Figs. 6a-c. In addition, good correlations were observed between the three relationships. These results indicate that a close relationship exists between the barrier function of the skin and skin permeation profiles.

Skin Permeation and Concentration of Lidocaine A marketed lidocaine patch was used following the PSA pretreatment. In vitro skin permeation techniques were also used to determine the skin permeation rate and concentration of lidocaine in the skin of hairless rats. Lidocaine permeation from the patch formulation was increased by stripping or peeling pretreatments of the stratum corneum using several PSAs. However, an adequate concentration of drugs in the skin is more important than an increase in skin permeation for the
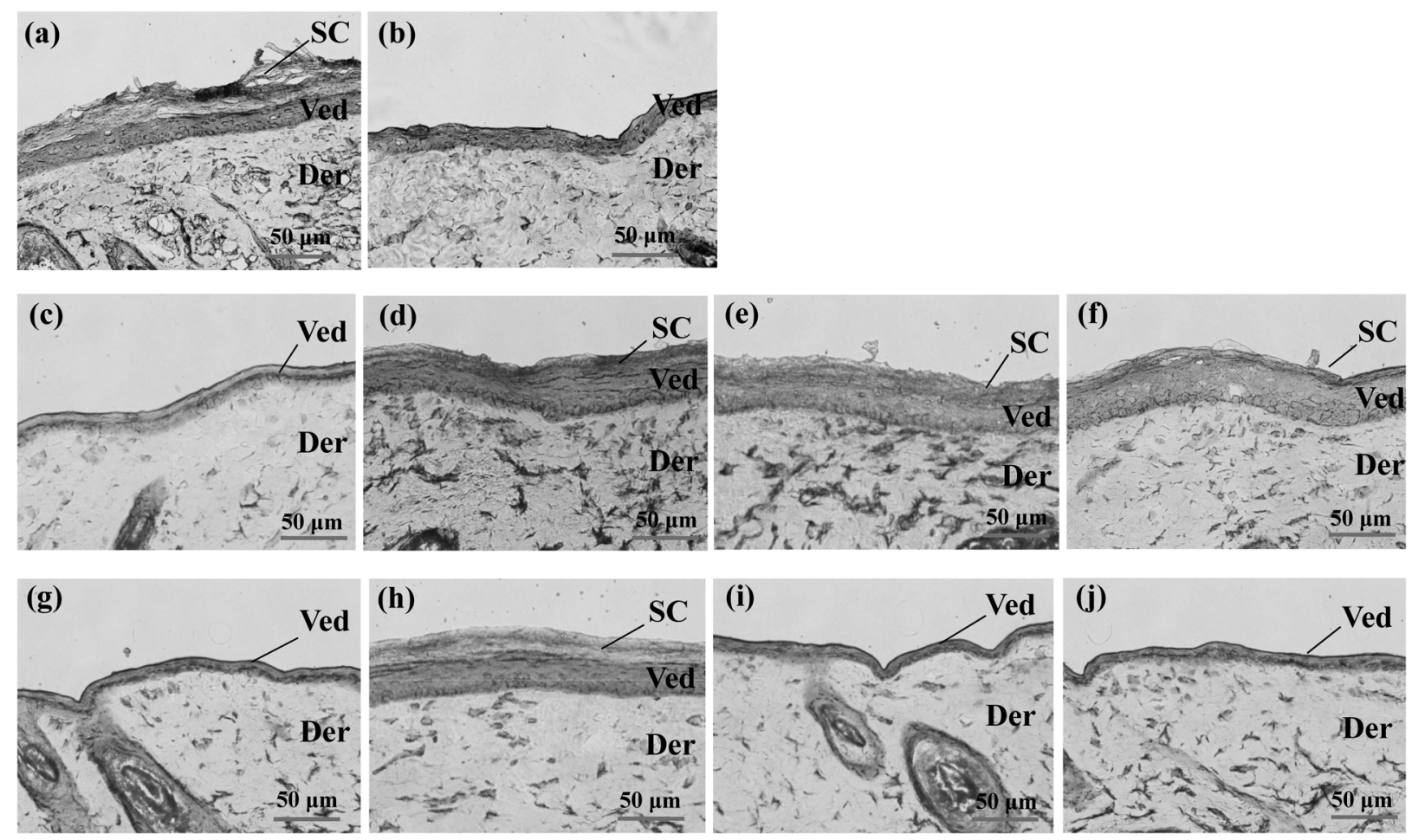

Fig. 4. H.E. Images of Hairless Rat Dorsal Skin Observed by a Phase Microscope

(a) Intact skin, (b) Skin stripped 20 times with Cellotape ${ }^{\circledR}$, (c)-(f) Stripped 5 times with PSA-A, -B, Cellotape ${ }^{\circledR}$, and Scotch Tape, respectively, and (g)-(j) Stripped 10 times with PSA-A, -B, Cellotape ${ }^{\mathbb{R}}$, and Scotch Tape, respectively 

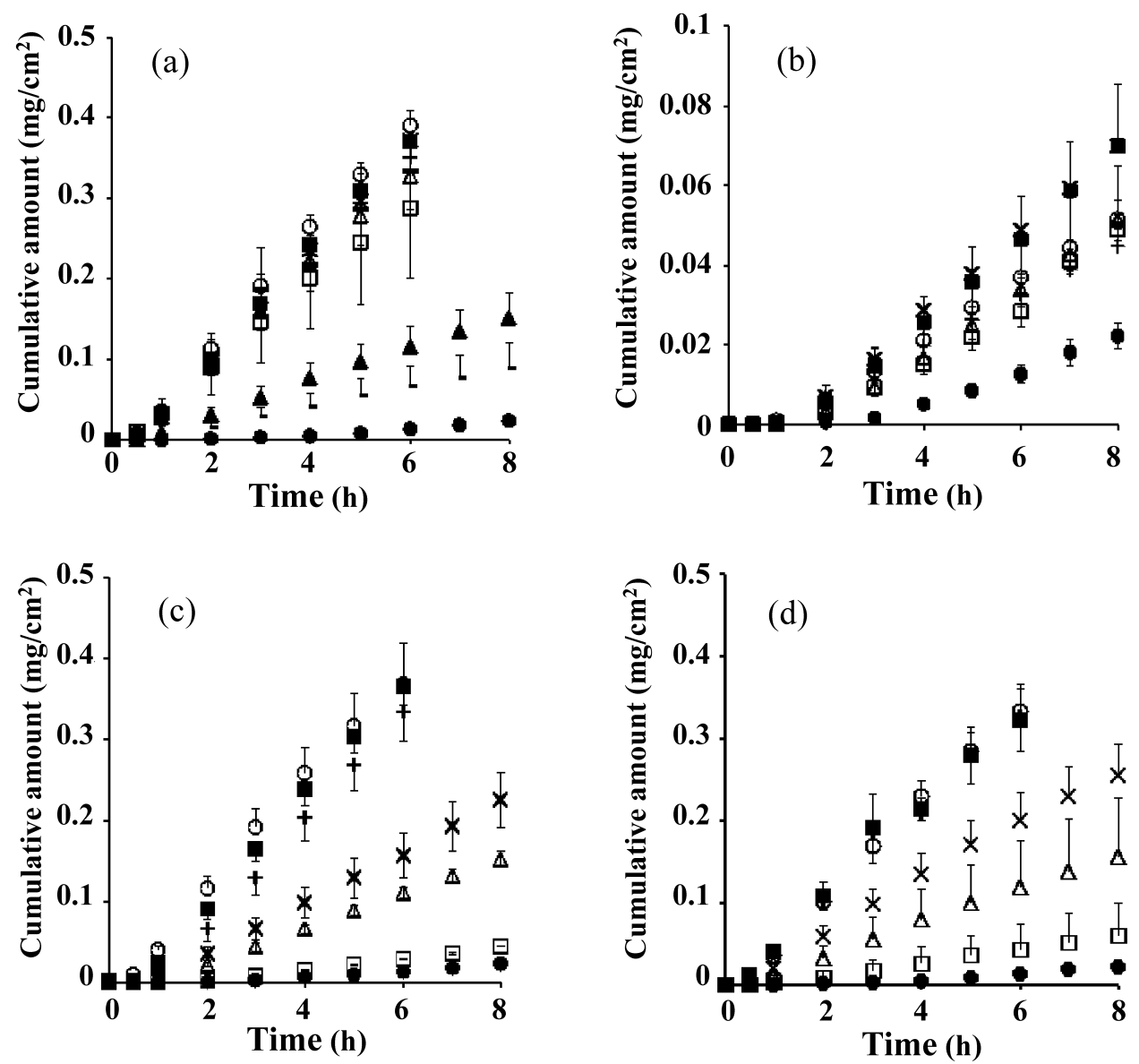

Fig. 5. Effect of the Number of Times Skin Was Stripped on the Cumulative Amount of Lidocaine That Permeated through Hairless Rat Skin

(a) PSA-A, (b) PSA-B, (c) Cellotape ${ }^{\circledR}$ and (d) Scotch Tape. Symbols: O: intact, $-: 1, \mathbf{\Delta}: 2, \square: 3,-: 4, \triangle: 5, \times: 7, \mathbf{0}: 10$, O: 15, +: 20. Each value shows the mean \pm S.E. $(n=3-6)$.

rapid onset of pharmacological effects in skin tissues in which topical formulations are applied. We then removed several layers of the stratum corneum using PSA-A or -B prior to the skin permeation experiments. The concentration of lidocaine was determined in the skin after the permeation experiments.

Figure 7 shows the effect of stripping the skin 5 times with PSA-A or -B on the skin permeation profiles of lidocaine from the patch formulation. The cumulative amount of lidocaine that permeated through skin that had been stripped 5 times with PSA-A over $3 \mathrm{~h}$ was very similar to that of skin stripped 20 times over $3 \mathrm{~h}$ with Cellotape. On the other hand, the cumulative amount of lidocaine that permeated through skin stripped 5 times with PSA-B over $8 \mathrm{~h}$ was approximately 3 times that through intact skin (without tape stripping). These results indicate that PSA-A and -B had different skin permeation-enhancing effects, similar to the results shown in Fig. 5.

Figure 8 shows the concentration of lidocaine in the dorsal skin following the in vitro skin permeation experiments. The concentration of lidocaine in skin that had been stripped 5 times with PSA-A was similar to that in skin stripped 20 times with Cellotape. In addition, these concentrations were significantly higher than those in intact skin (34.5 fold, $p<0.01)$. On the other hand, the concentration of lidocaine in skin stripped five times with PSA-B was 4.4-fold higher than that in intact skin $(p<0.05)$, but was markedly lower than that in skin stripped 20 times with Cellotape.

Figure 9 shows the concentration of lidocaine in skin pre- treated with different PSAs 10 or 30 min after the application of lidocaine tape. Stripping a few times with PSA-A markedly increased the concentration of lidocaine in the skin. In contrast, stripping one time with PSA-B resulted in a similar skin concentration $30 \mathrm{~min}$ after the application of lidocaine patch. Almost the same level of concentration lidocaine was found in the skin even after stripping had been performed more times. In contrast, the skin level of lidocaine gradually increased until the skin had been stripped 5 times with Cellotape.

\section{Discussion}

The $180^{\circ}$ peel, probe tack, and ball tack methods were used in the present study to determine the adhesive force of PSAs. It is difficult to conclude which is the best marker for determining the adhesive force because each method resulted in a different order. However, we considered PSA-B to have had the lowest adhesive force, whereas Scotch Tape had the highest among the 4 PSAs tested in this study (Table 2). The adhesive force of PSA-A was higher than that of PSA-B, but was lower than that of Scotch Tape (Table 2).

Interestingly, the amount of the stratum corneum removed from hairless rat dorsal skin by the first stripping procedure was in the order of PSA-A $>$ PSA-B $>$ Scotch Tape $=$ Cellotape (Fig. 1). This order was not similar to that observed in the adhesive test as shown in Table 2. Thus, it is also difficult to determine the relationship between the adhesive forces obtained by the $180^{\circ}$ peel, probe tack, and ball tack methods and the 

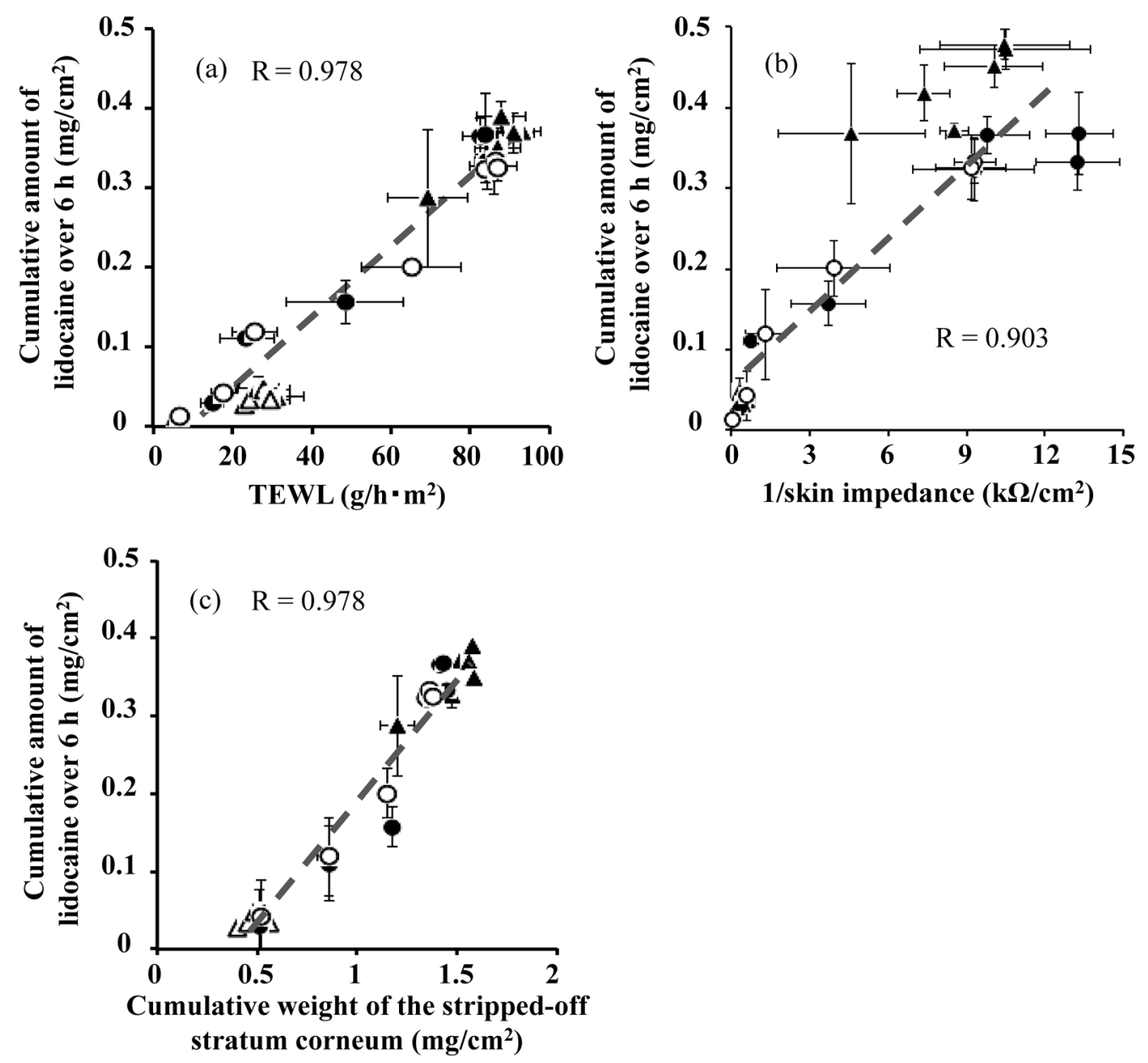

Fig. 6. Effect of Skin Barrier Functions; TEWL (a), Reverse of Skin Impedance (b) and Cumulative Weight of the Stripped Stratum Corneum on the Cumulative Amount of Lidocaine That Permeated through Hairless Rat Skin over $6 \mathrm{~h}$

Symbols: $\mathbf{\Delta}$ : PSA-A, $\triangle$ : PSA-B, $\bullet$ : Cellotape ${ }^{\circledR}$, O: Scotch Tape. Each value shows the mean \pm S.E. $(n=3-6)$.

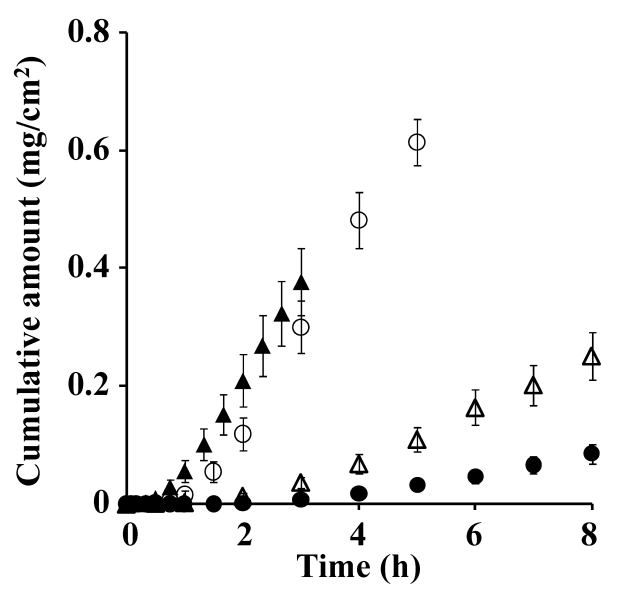

Fig. 7. Time Course of the Cumulative Amount of Lidocaine Permeated from the Marketed Patch through Hairless Rat Intact Dorsal Skin, Stripped 20 Times with Cellotape and 5 Times with PSA-A and -B

Symbols: $\boldsymbol{\Delta}$ : stripped 5 times with PSA-A, $\triangle$ : stripped 5 times with PSA-B, $\mathbf{0}$ intact skin, $\bigcirc$ : stripped 20 times with Cellotape. Each value shows the mean \pm S.E. $(n=3-4)$.

weight of the stratum corneum removed by first strip.

PSA-A generally exhibited a higher peeling capacity than the two marketed PSAs (Fig. 2). Interestingly, stripping skin two or three times with PSA-A or ten times with the marketed

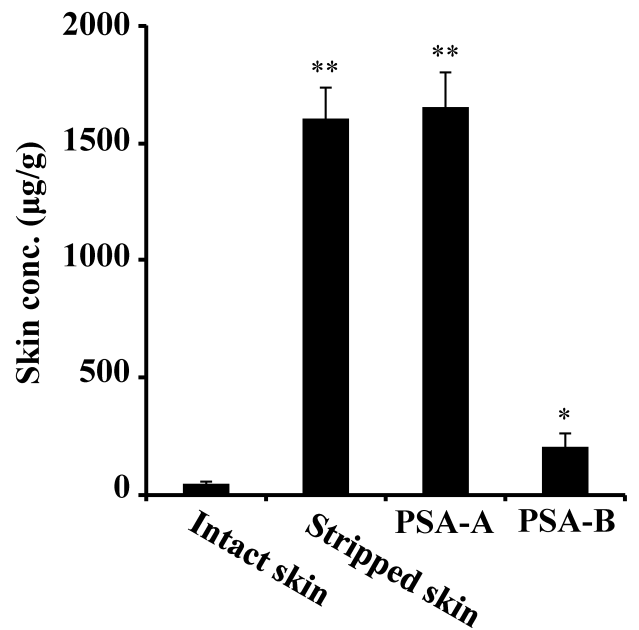

Fig. 8. Lidocaine Concentration in Skin Stripped 20 or 5 Times with Cellotape or PSA-A and -B, Respectively, after Application of the Marketed Lidocaine Patch

Each value shows the mean \pm S.E. $(n=3)$. Statistical analysis was performed by ANOVA with nonrepeated measures. ${ }^{*} p<0.05,{ }^{*} * p<0.01$.

PSAs removed a similar amount of the stratum corneum from the skin, which suggested that stripping only a few times with PSA-A is very useful for decreasing the skin barrier in the stratum corneum. In contrast, stripping skin two or 


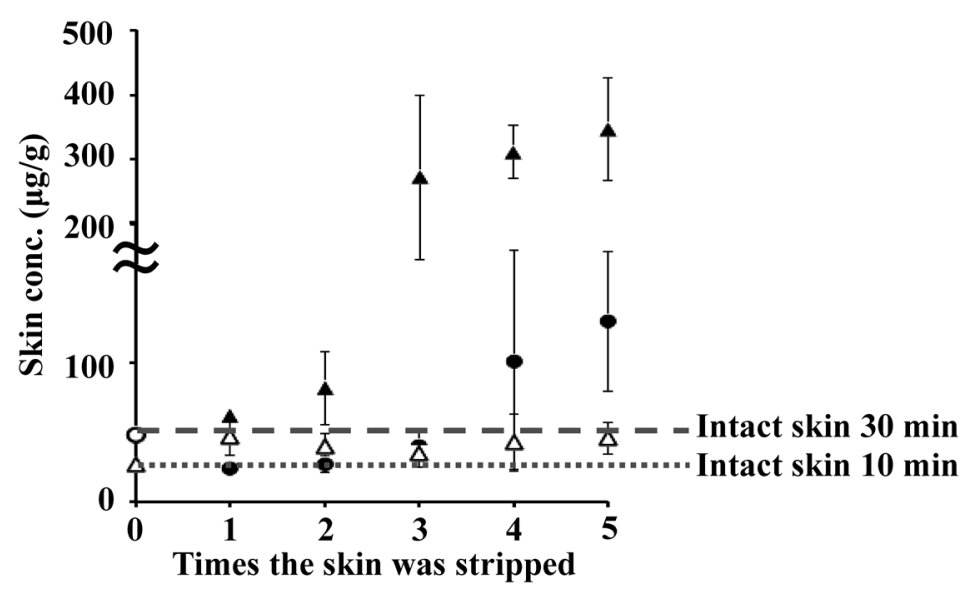

Fig. 9. Effect of the Number of Times Skin Was Stripped on the Skin Concentration of Lidocaine after a 10-min Application of the Marketed Lidocaine Patch

Symbols: $\Delta$ : PSA-A, $\triangle$ : PSA-B, $\bullet$ : Cellotape ${ }^{\circledR}$. Each value shows the mean \pm S.E. $(n=3)$.

more times with PSA-B did not remove a large amount of the stratum corneum. Thus, PSA-B can be used as a safe peeling material to increase the skin permeation of drugs as well as remove several layers of the stratum corneum. Lactic acid and glycolic acid have already been used as peeling materials for several layers of the stratum corneum. PSA-B may also be used as an alternative to these acids to treat skin dullness or somberness treatment especially in women, which is one of the largest complaints in facial beauty.

The amount of the stratum corneum removed by PSAs from hairless rat dorsal skin was approximately twice that removed from pig ear skin (Fig. 1). A good correlation $\left(R^{2}=0.995\right)$ was observed between the amount of the stratum corneum removed from rat skin and pig skin (data not shown), which suggests that the stratum corneum in pig skin is very tight, similar to human skin, and that the stratum corneum in rat skin is markedly looser than that in pig skin and human skin. The difference in the amount of the stratum corneum removed by PSAs between pig skin and hairless rat skin may be useful for estimating the skin permeation profiles of chemical compounds through the stripped skin of both species.

The amount of the stratum corneum removed from PSAs (Fig. 2) was closely correlated to TEWL and the reciprocal of skin impedance (Fig. 3). In addition, these profiles were supported by morphological observations of skin slices as shown in Fig. 4. The skin permeation of lidocaine (Fig. 5) was also shown to be related to these results in Figs. 2, 3, and 4, as shown in Fig. 6. However, the results shown in Figs. 2-6 did not correlate well with their adhesive forces (Table 2). These results may be due to different surface morphologies between the test boards or balls used to determine the adhesive forces of PSAs on the stratum corneum. The surface and deep layers of the stratum corneum have different properties, which have also implicated in the results obtained in Figs. 2 and 3. Further experiments may be necessary to obtain an artificial stratum corneum surface to mimic the in vivo adhesive forces of PSAs.

The results shown in Figs. 7, 8, and 9 suggest that stripping the stratum corneum three times with PSA-A is sufficient to increase the skin permeation and concentration of lidocaine following its application in the marketed lidocaine patch. Thus, PSA-A can be used as a pretreatment material prior to the application of transdermal therapeutic systems and topical drug formulations. In contrast, PSA-B can be very safely utilized to peel the stratum corneum because the skin barrier function remained relatively constant with the number of strips performed. Many researchers have reported the relationship between skin permeation of drugs and the reciprocal value of TEWL. ${ }^{21)}$ Thus, human skin permeation of drugs would be predicted by the TEWL value after PSA treatment.

\section{Conclusion}

Pretreating skin with PSA-A, in particular, can be used to markedly enhance skin permeation; the enhancement ratio of the skin permeation of lidocaine was approximately 22 times higher than that through intact skin. PSA-A can also be used to increase the skin concentration of topically applied chemical compounds. The pretreatment of skin with PSA-B can be used to safely increase the skin permeation and skin concentration of drugs, although its enhancement effect was not as high as that with PSA-A.

In conclusion, PSA-A and -B exhibited different abilities to increase the skin permeation and skin concentration of drugs. Considering their different penetration-enhancing effects, PSA-A and -B can be utilized as a pretreatment material and safe peeling material, respectively.

Acknowledgment We appreciated Mr. Tomio Hatanaka, Lintec Corporation for his technical advice and assistance.

\section{References}

1) Ooi K., Sasaki H., Sugawa T., Kimura S., Ueda H., Numajiri S., Kojima S., Katayama T., Mizutani Y., Morimoto Y., J. Pharm. Health Care Sci., 34, 1011-1016 (2008).

2) Barry B. W., "Dermatological Formulations: Percutaneous Absorption," Marcel Dekker, New York, 1983.

3) Blank I. H., J. Invest. Dermatol., 45, 249-256 (1965).

4) Moser K., Hriwet T., Nail A., Guy R. H., Eur. J. Pharm. Biopharm., 52, 103-112 (2001).

5) Sugibayashi K., Oshizaka T., Todo H., Fragrance J, 38, 53-60 (2010).

6) Sugibayashi K., J. Soc. Cosmet. Chem. Japan, 41, 241-245 (2007).

7) Wu X. M., Todo H., Sugibayashi K., Int. J. Pharm., 316, 102-108 (2006).

8) Yan K., Todo H., Sugibayashi K., Int. J. Pharm., 397, 77-83 (2010). 
9) Wu X. M., Todo H., Sugibayashi K., J. Controlled Release, 118, 189-195 (2007).

10) Tokudome Y., Sugibayashi K., J. Controlled Release, 92, 93-101 (2003).

11) Tokudome Y., Sugibayashi K., J. Controlled Release, 95, 267-274 (2004).

12) Tokumoto S., Mori K., Higo N., Sugibayashi K., J. Controlled Release, 105, 296-304 (2005).

13) Japanese Industrial Standards: Adhesive tape and sheet test, JIS Z0237 (2009).

14) Breternitz M., Flach M., Elsner P., Fluhr J. W., Br. J. Dermatol., 156, 231-240 (2007).
15) Okumura M., Sugibayashi K., Ogawa K., Morimoto Y., Chem. Pharm. Bull., 37, 1404-1406 (1989).

16) Yamada K., Yamashita J., Todo H., Hashimoto S., Tokudome Y., Hashimoto F., Sugibayashi K., J. Oleo Sci., 60, 31-40 (2011).

17) Jaymin C. S., Int. J. Pharm., 90, 161-169 (1993).

18) Scheuplein R. J., J. Invest. Dermatol., 48, 79-88 (1967).

19) Potts R. O., Guy R. H., Pharm. Res., 9, 663-669 (1992).

20) Yukawa J., Sugibayashi K., Morimoto Y., J. Pharm. Sci. Technol. Jpn., 49, 254-262 (1989).

21) Herkenne C., Alberti I., Naik A., Kalia Y. N., Mathy F. X., Preat V., Guy R. H., Pharm. Res., 25, 87-103 (2007). 